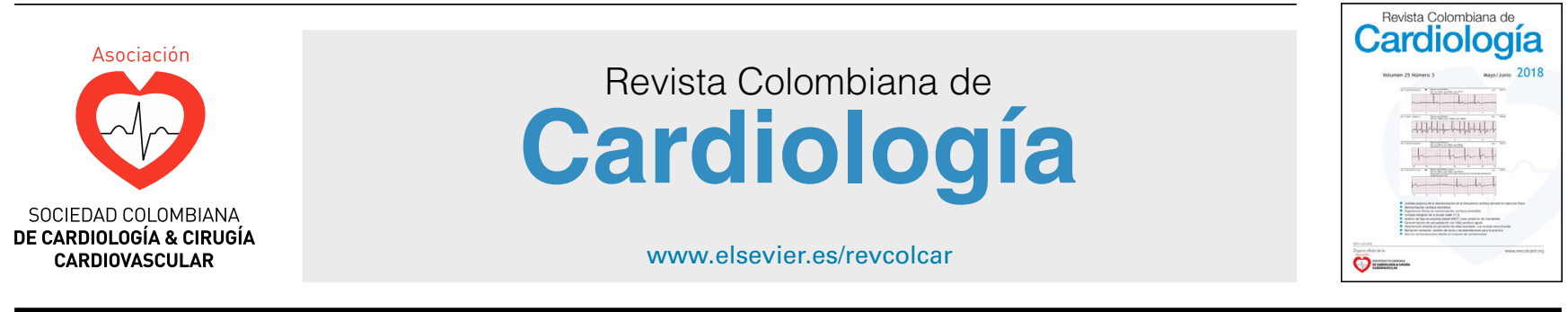

CARDIOLOGÍA DEL ADULTO - ARTÍCULO ORIGINAL

\title{
Bostezo como mecanismo protector y predictor de síncope: estudio observacional en una cohorte de pacientes
}

\author{
Mauricio Duque*, Omar Cortés, Laura Duque, María C. Gaviria, \\ Elsa M. Vásquez-Trespalacios, Juan C. Díaz-Martínez, Jorge E. Marín, \\ Julián M. Aristizábal, Jorge E. Velásquez y William Uribe
}

\author{
Departamento de Cardiología y Electrofisiología, Universidad CES, Clínica CES, CES Cardiología, Medellín, Colombia
}

Recibido el 14 de julio de 2017; aceptado el 12 de junio de 2018

Disponible en Internet el 5 de diciembre de 2018

\author{
PALABRAS CLAVE \\ Cardiología; \\ Síncope; \\ Sistema nervioso \\ autónomo
}

\begin{abstract}
Resumen
Introducción: el bostezo se presenta con frecuencia en los pacientes sometidos a prueba de mesa basculante. Estudios previos han demostrado que el bostezo estimula el sistema nervioso simpático con sus consecuentes cambios hemodinámicos, principalmente en la frecuencia cardíaca y la presión arterial.

Objetivo: describir los efectos hemodinámicos y sobre el sistema nervioso autónomo del bostezo, así como establecer el bostezo como mecanismo protector de síncope o predictor de respuesta hemodinámica positiva.

Métodos: estudio observacional de fuente secundaria, realizado a partir de una base de datos de pacientes que fueron sometidos a prueba de mesa basculante entre agosto de 2010 hasta diciembre de 2015 y presentaron al menos un bostezo durante la prueba. Se realizaron análisis estadísticos y pruebas pareadas para determinar correlaciones.

Resultados: se incluyeron 156 pacientes, la mayoría mujeres, edad promedio 40 años.

43 pacientes presentaron síncope, 113 (72,4\%) bostezo sin síncope, y de éstos, 50 (44,2\%) tuvieron bostezo y prueba de mesa basculante con respuesta positiva. Se observó un aumento en la mayoría de variables hemodinámicas, sin embargo, hubo una disminución significativa de la frecuencia cardíaca. En 67 pacientes se observó bostezo como protector de síncope mientras que en 61 pacientes como predictor de respuesta hemodinámica positiva.

Conclusión: el bostezo se asoció con activación del sistema nervioso simpático, con efectos hemodinámicos y sobre el sistema nervioso autónomo. Puede plantearse como un signo predictor de respuesta hemodinámica positiva de tipo mixto o vasodepresora y en otros casos como un mecanismo protector frente a la aparición de síncope.

(C) 2018 Sociedad Colombiana de Cardiología y Cirugía Cardiovascular. Publicado por Elsevier España, S.L.U. Este es un artículo Open Access bajo la licencia CC BY-NC-ND (http:// creativecommons.org/licenses/by-nc-nd/4.0/).
\end{abstract}

\footnotetext{
* Autor para correspondencia.

Correo electrónico: mauricioduquemd@gmail.com (M. Duque).
} 


\section{KEYWORDS}

Cardiology;

Syncope;

Autonomic nervous

system
Yawning as a protective mechanism and predictor of syncope: observational study in a patient cohort

\begin{abstract}
Introduction: Yawning is often present in patients subjected to a tilt table test. Previous studies have demonstrated that yawning stimulates the sympathetic nervous system, with its subsequent haemodynamic changes, mainly heart rate and blood pressure.

Objective: To describe the haemodynamic effects of yawning on the autonomic nervous system, as well as to establish yawning as a protective mechanism of syncope, or a predictor of a positive haemodynamic response.

Methods: A secondary source observational study conducted using a database of patients that were subjected to the tilt table test between August 2010 and December 2015, and yawned at least once during the test. Statistical analyses and paired tests were performed to determine correlations.

Results: A total of 156 patients were included, the majority women, and a mean age of 40 years. Syncope was presented in 43 patients. The remaining $113(72.4 \%)$ yawned with no syncope, and of these, $50(44.2 \%)$ had yawned and had a positive response to the tilt-table test. Although there was an increase in the majority of haemodynamic variables, a significant decrease was observed in the heart rate. Yawning was observed as a protector of syncope in 67 patients, whilst it was a predictor of a positive haemodynamic response in 61 patients.

Conclusion: Yawning is associated with activation of the sympathetic nervous system, with haemodynamic effects and on the autonomic nervous system. It could be established as a predictive sign of a mixed type or vasodepressor positive haemodynamic response, and in other cases as a protective mechanism against the appearance of a syncope.

(c) 2018 Sociedad Colombiana de Cardiología y Cirugía Cardiovascular. Published by Elsevier España, S.L.U. This is an open access article under the CC BY-NC-ND license (http:// creativecommons.org/licenses/by-nc-nd/4.0/).
\end{abstract}

\section{Introducción}

El síncope es una pérdida súbita y transitoria de la conciencia, de corta duración y recuperación espontánea, secundario a una hipoperfusión cerebral global. Es una causa común de visita a los servicios de urgencias y de consulta externa ${ }^{1}$. Dentro de sus diferentes etiologías, el síncope neuralmente mediado es considerado la causa más frecuente ${ }^{2}$.

Con el advenimiento de equipos que permiten la monitorización continua y en tiempo real de variables hemodinámicas y del sistema nervioso autónomo, se desarrolló la prueba de mesa basculante, herramienta que, por medio de un escenario controlado y un protocolo establecido, impone un reto ortostático para evaluar el comportamiento del sistema nervioso autónomo tanto simpático como parasimpático. Sofisticados equipos para la realización de dicha prueba permiten monitorizar variables como: presión arterial, índice cardíaco, resistencia vascular periférica, frecuencia cardíaca, variabilidad de la frecuencia cardíaca, además de la posibilidad de generar análisis espectral basado en dominios donde el dominio de alta frecuencia (HF, su sigla en inglés), se relaciona con el sistema nervioso parasimpático y el de baja frecuencia (LF, su sigla en inglés) con el sistema nervioso simpático y parasimpático. La relación baja frecuencia sobre alta frecuencia (LF/HF) se considera un reflejo más preciso del balance simpático vagal $^{3,4}$.
Los principales síntomas referidos por los pacientes durante la prueba de mesa basculante son cefalea, suspiros, parestesias, sensación de calor, palpitaciones, náuseas e hiperventilación. Se ha considerado que dichos síntomas sirven como medidas contrarregulatorias o premonitorias frente al síncope. En el laboratorio de neurocardiología se ha observado con cierta frecuencia la aparición de bostezos y su relación con efectos hemodinámicos durante las diferentes fases de la prueba. En ocasiones, el bostezo aparece previo al desarrollo de la respuesta hemodinámica y en otras de forma más tardía, lo que sugiere que se comporta como un mecanismo anticipatorio o protector frente a la aparición de síncope. En consecuencia, investigaciones han relacionado al bostezo con diferentes efectos hemodinámicos sobre la frecuencia cardíaca, la presión arterial, la frecuencia respiratoria y el volumen tidal, y han demostrado que ejerce una activación del sistema nervioso simpático reflejado principalmente en los primeros 10 segundos posteriores a éste ${ }^{5,6}$.

En la literatura revisada por los autores no se encontró evidencia de una relación entre el botezo y el síncope o respuesta hemodinámica positiva durante la prueba de mesa basculante y la aparición o prevención del síncope. Tampoco se ha utilizado la cardioimpedancia con medición en tiempo real de variables hemodinámicas y del sistema nervioso autónomo para hacer una correlación directa entre el bostezo y su relación con el sistema nervioso autónomo y el sistema cardiovascular, por tanto, el objetivo de esta investigación fue describir los efectos hemodinámicos más frecuentes del bostezo y su influencia sobre las variables 
autonómicas para así determinar si es un mecanismo protector o predictor de síncope o respuesta positiva en pacientes llevados a prueba de mesa basculante por sospecha de síncope neuralmente mediado.

\section{Materiales y métodos}

Estudio observacional, de fuente secundaria, realizado a partir de una base de datos del Servicio de Cardiología y Electrofisiología del grupo CES Cardiología de la ciudad de Medellín, Colombia, en el que se incluyeron registros de pacientes entre el 17 de agosto de 2010 hasta el 02 de diciembre de 2015, que se sometieron a prueba de mesa basculante con base en el protocolo italiano ${ }^{7}$ y en el equipo Task Force Monitor 3040i cnsystems.

Cada una de las pruebas fue interpretada por un cadiólogo electrofisiólogo y se clasificó la respuesta de acuerdo con lo establecido por el VASIS 5. Cada paciente incluido en el estudio presentó mínimo un episodio de bostezo.

Se diseñó un cuestionario para completar las variables extraídas del registro del equipo: frecuencia cardíaca, presión arterial, índice cardíaco, resistencia vascular periférica y variabilidad de la frecuencia cardíaca de acuerdo con lo propuesto por la Sociedad del Ritmo Cardíaco ${ }^{8}$ : LF, HF y relación LF/HF, y su asociación en tiempo real con diferentes tomas de presión arterial sistólica respecto al bostezo: antes, durante, 5, 10 y 15 segundos después. La descripción univariada se realizó a través de las medidas de tendencia central y dispersión para las variables cuantitativas y con frecuencias relativas para los datos cualitativos. La comparación de los grupos antes, durante y después del bostezo y antes, durante y después del síncope, se hizo por medio de pruebas pareadas. El nivel de significancia de las pruebas de hipótesis se estableció en 0,05. También se analizaron estas mismas variables respecto al síncope. Los intervalos de tiempo se escogieron con base en estudios previos que han demostrado que los efectos de este mecanismo no duran más de 15 segundos después de su inicio, y han utilizado también intervalos de 5 segundos para evaluar los cambios asociados 9 .

\section{Resultados}

Se incluyeron 156 pacientes en quienes se documentó mínimo un episodio de bostezo durante la prueba de mesa basculante. 108 fueron mujeres, 25\% de las cuales presentó síncope y el $56,4 \%$ prueba de mesa basculante con respuesta hemodinámica positiva. De 48 hombres, 33,3\% presentó síncope y $66,7 \%$ prueba de mesa basculante con respuesta hemodinámica positiva. En total, 93 pacientes $(59,6 \%)$ tuvieron prueba de mesa basculante positiva. Las características demográficas se detallan en la tabla 1.

El promedio de tiempo durante el cual se presentaron los bostezos fue al minuto 34,33 con un mínimo de 11,6 minutos; en algunos casos fue posterior a la finalización de la fase de inclinación durante la recuperación. Al analizar la relación entre la aparición de bostezo y la administración de nitroglicerina, se encontró que la mayoría de los episodios ocurrió luego de la administración del medicamento como parte del protocolo del prueba de mesa basculante a los 27 minutos de iniciado (65,3\%; 102 casos). En el análisis por subgrupos,
Tabla 1 Características demográficas de la muestra

\begin{tabular}{ll}
\hline Característica & $\mathrm{n}(\%)$ \\
\hline $\begin{array}{l}\text { Edad-años } \\
\quad \text { (promedio } \pm \mathrm{DS})\end{array}$ & \\
$\begin{array}{l}\text { Género } \\
\text { Femenino }\end{array}$ & $40,07+/-21,11$ \\
$\quad$ Masculino & $108(69,2 \%)$ \\
Pacientes con síncope & $48(30,8 \%)$ \\
$\quad$ prueba de mesa basculante positiva) & $43(27,6 \%)$ \\
Mujeres con síncope & $27(25 \%)$ \\
Hombres con síncope & $16(33 \%)$ \\
Pacientes sin síncope & $113(72,4 \%)$ \\
Prueba de mesa basculante positiva & $50(32 \%)$ \\
Prueba de mesa basculante negativa & $63(40,3 \%)$ \\
Prueba de mesa basculante positiva & $93(59,6 \%)$ \\
$\quad$ Respuesta tipo I o mixta & $33(35,5 \%)$ \\
$\quad$ Respuesta tipo II o cardioinhibitoria & $0(0 \%)$ \\
Respuesta tipo II o vasodepresora & $60(64,5 \%)$ \\
\hline
\end{tabular}

se encontró que en el grupo sin síncope la prevalencia de bostezo posterior y anterior a la nitroglicerina fue de $72,5 \%$ y $27,4 \%$, respectivamente. En el grupo de pacientes con síncope la prevalencia de bostezo después de la nitroglicerina disminuyó a 46,5\%, mientras que antes de la nitroglicerina aumentó a $53,4 \%$.

\section{Variables hemodinámicas y del sistema autónomo}

Respecto a las variables hemodinámicas y del sistema autónomo, sus frecuencias, valores mínimos y máximos encontrados se muestran en la tabla 2. Se hicieron pruebas pareadas entre momentos antes, durante y después del bostezo para las variables de mayor relevancia hemodinámica (tablas 3 a 5).

El índice cardíaco presentó una disminución estadísticamente significativa durante el inicio del bostezo, explicado probablemente por la disminución de la frecuencia cardíaca asociada al aumento en la resistencia vascular periférica. Esta última aumentó de manera significativa durante el bostezo y 10 a 15 segundos después, al compararlo con la resistencia vascular periférica antes del episodio. Así mismo, fue significativamente mayor antes del síncope y presentó una disminución importante durante el síncope e incluso persistió baja en la recuperación, hallazgo de esperarse debido a que las respuestas del prueba de mesa basculante fueron todas de tipo mixto y vasodepresoras.

En la mayoría de pacientes hubo una disminución significativa de la frecuencia cardíaca, con un promedio de 13,7 latidos por minuto (deviación estándar -DE- antes de bostezo 21,56 vs. DE del máximo cambio 10,99; $p<0,001$ ), alcanzando un valor máximo de 49 lpm, cambio mayor especialmente a los 5 y 10 segundos posteriores al inicio del bostezo (el $29,2 \%$ ocurrió a los 5 segundos y el $24,8 \%$ a los 10 segundos).

Tanto la presión arterial sistólica como la diastólica aumentaron de manera significativa en la mayoría de pacientes, principalmente la sistólica, en la cual el incremento 
Tabla 2 Variables hemodinámicas de la muestra

\begin{tabular}{|c|c|c|c|c|c|}
\hline Característica & $\mathrm{n}$ & Mínimo & Máximo & Promedio & $\mathrm{DE}(\mathrm{DE})$ \\
\hline Frecuencia cardíaca inicial (lpm) & 156 & 40 & 124 & 66,54 & 13,176 \\
\hline PA inicial (mm Hg) & 154 & 0 & 0 & 0 & 0 \\
\hline GC inicial & 153 & 2,2 & 11,3 & 5,065 & 1,46 \\
\hline IC inicial & 153 & 1,3 & 6 & 3,011 & 0,927 \\
\hline RVP inicial & 153 & 725 & 3334 & 1498,63 & 532,2 \\
\hline LF inicial $(\mathrm{m} / \mathrm{s})$ & 155 & 1 & 13176 & 1245,5 & 1819,48 \\
\hline HF inicial (m/s) & 155 & 2 & 19288 & 1967,04 & 3259,42 \\
\hline LF/HF inicial & 155 & 0 & 60 & 2,185 & 5,296 \\
\hline Minuto del bostezo & 136 & 11,6 & 52,4 & 34,33 & 7,19 \\
\hline \multicolumn{6}{|l|}{ Máximo } \\
\hline $\begin{array}{l}\text { cambio de frecuencia } \\
\text { cardíaca (lpm) }\end{array}$ & 121 & 1 & 49 & 16,86 & 9,88 \\
\hline IC antes bostezo & 149 & 1,5 & 37 & 3,128 & 3,689 \\
\hline RVP antes bostezo & 149 & 609 & 2878 & 1452,59 & 443,05 \\
\hline LF antes bostezo & 155 & 2 & 8208 & 714,34 & 1167,74 \\
\hline HF antes bostezo & 155 & 1 & 10642 & 403,12 & 1238,54 \\
\hline LF/HF antes bostezo & 154 & 0,1 & 59 & 5,8 & 8,47 \\
\hline IC durante bostezo & 149 & 1,2 & 4,8 & 2,57 & 0,66 \\
\hline Resistencia vascular periférica durante bostezo & 149 & 619 & 3030 & 1566,81 & 496,61 \\
\hline LF durante bostezo & 154 & 3 & 23861 & 1038,71 & 2173,8 \\
\hline HF durante bostezo & 154 & 1 & 10015 & 405,36 & 1191,25 \\
\hline LF/HF durante bostezo & 153 & 0,1 & 110 & 9,267 & 14,73 \\
\hline LF antes de síncope o RTA HF antes de & 43 & 1 & 8526 & 1087,37 & 1897,93 \\
\hline síncope o RTA & 43 & 3 & 4073 & 514,42 & 935,417 \\
\hline
\end{tabular}

GC: gasto cardiaco; HF: alta frecuencia; IC: índice cardiaco; LF: baja frecuencia; RVP: resistencia vascular periférica.

Tabla 3 Variables hemodinámicas antes versus durante el bostezo

\begin{tabular}{llll}
\hline Variable & Antes & Durante & Valor de P \\
\hline IC $\left(\mathrm{L} / \mathrm{min} / \mathrm{m}^{2}\right)$ & 3,12 & 2,56 & 0,059 \\
Resistencia vascular periférica (dinas.s.cm-5) & 1452 & 1566 & 0,0001 \\
LF $\left(\mathrm{ms}^{2}\right)$ & 718 & 1038 & 0,028 \\
HF $\left(\mathrm{ms}^{2}\right)$ & 405,56 & 406,36 & 0,98 \\
LF $/ \mathrm{HF}(\%)$ & 5,87 & 9,81 & 0,004 \\
\hline
\end{tabular}

Tabla 4 Variables hemodinámicas antes versus después del bostezo

\begin{tabular}{llll}
\hline Variable & Antes & Después & Valor de $\mathrm{p}$ \\
\hline IC $\left(\mathrm{L} / \mathrm{min} / \mathrm{m}^{2}\right)$ & 3,12 & 2,6 & 0,075 \\
Resistencia vascular periférica (dinas.s.cm-5) & 1452 & 1529 & 0,001 \\
LF $\left(\mathrm{ms}^{2}\right)$ & 718 & 774 & 0,24 \\
HF $\left(\mathrm{ms}^{2}\right)$ & 403,12 & 413,88 & 0,6 \\
LF/HF $(\%)$ & 5,83 & 6,61 & 0,36 \\
\hline
\end{tabular}

Tabla 5 Variables hemodinámicas durante versus después del bostezo

\begin{tabular}{llll}
\hline Variable & Durante & Después & Valor de $\mathrm{p}$ \\
\hline re $\left(\operatorname{Limin} / \mathrm{m}^{2}\right)$ & 2,56 & 2,6 & 0,19 \\
resistencia vascular periférica (dinas.s.cm-5) & 1566 & 1529 & 0,148 \\
LF $\left(\mathrm{ms}^{2}\right)$ & 1044 & 779 & 0,05 \\
HF $\left(\mathrm{ms}^{2}\right)$ & 405,36 & 416,11 & 0,4 \\
\hline
\end{tabular}


máximo promedio que se alcanzó fue de $12,7 \mathrm{~mm} \mathrm{Hg}$ (DE antes del bostezo 18,7 vs. DE del máximo cambio 10,7; $\mathrm{p}<0,001)$, pero se llegó hasta valores de $69 \mathrm{~mm} \mathrm{Hg}$. La presión arterial diastólica aumentó en promedio $7,03 \mathrm{~mm}$ $\mathrm{Hg}$ (DE antes del bostezo 14,3 vs. DE del máximo cambio $6,1 ; \mathrm{p}<0,001)$, con valor máximo de $25 \mathrm{~mm} \mathrm{Hg}$; en ambos casos el momento del máximo cambio se repartió de manera similar en el inicio del bostezo (presión arterial sistólica el $21,2 \%$, diastólica el 20,4\%), 5 segundos posteriores (presión arterial sistólica el $20,4 \%$, diastólica el $22,1 \%$ ) y 10 segundos después (presión arterial sistólica el 22,1\%, diastólica $20,4 \%)$.

Se encontró un aumento significativo de la relación LF/HF durante el momento del bostezo con disminución después de los 15 segundos de ocurrido el evento, aunque a niveles ligeramente superiores a los obtenidos previamente. De esta manera se refleja una activación importante del simpático en respuesta al bostezo, con una tendencia a mantenerse posterior a éste (DE antes 8,47 vs. DE durante 16,2; $p$ 0,004)

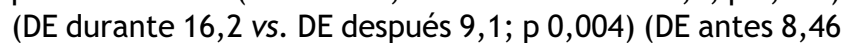
vs. DE después 9,$1 ; p 0,36)$.

Al evaluar la relación LF/HF en los pacientes que presentaron síncope (alrededor del evento, antes, durante y en la recuperación, se observó que la relación LF/HF se correlacionaba con episodios sincopales, y al compararla con otros momentos del prueba de mesa basculante como antes y despues (DE 8,47 antes del bostezo o DE 9,1 después del bostezo), lo cual refleja el comportamiento del sistema nervioso autónomo durante las diferentes etapas del test de mesa basculante. Lo anterior se asoció a una caída de la presión arterial sistólica durante la prueba de mesa basculante, explicada por agotamiento progresivo de los mecanismos compensatorios y la caída de la presión arterial sistólica por un retiro abrupto del simpático y por estimulación súbita del estímulo parasimpático; se demostró que en el momento del síncope había una mayor disminución de la relación $\mathrm{LF} / \mathrm{HF}$, con una $\mathrm{DE}$ de 3,48 pero que no alcanzó significancia estadística (DE antes 5,41 vs. DE durante el síncope 3,48 ; p 0,071) reflejando el retiro simpático y mayor predominio parasimpático que ocurre durante el síncope.

La relación LF/HF fue menor en la fase de recuperación al compararla antes y durante el síncope, lo cual puede deberse a que en esta última fase el paciente ya se encuentra en decúbito supino y el reto ortostático ha cesado, mostrando un predominio vagal.

\section{Bostezo como protector de síncope}

En cuanto al bostezo como protector de síncope (casos en los cuales logró abortarlo), se presentó en 61 pacientes (54\% del grupo sin síncope y $39,1 \%$ del total de la muestra, incluidos aquellos que tuvieron síncope). En consecuencia, del total de pacientes que bostezaron casi el $40 \%$ empezaron a presentar caída de la presión arterial y síntomas como palpitaciones, náuseas, mareo, bostezo, entre otros. Al bostezar lograron un aumento significativo de la presión arterial, la resistencia vascular periférica y en la relación LF/HF, que evitó el síncope.

\section{Bostezo como predictor de respuesta}

En cuanto al bostezo como equivalente o predictor de respuesta (prueba de mesa basculante con respuesta hemodinámica positiva), se presentó en 67 pacientes, para un $59,2 \%$ del grupo sin síncope, y $43 \%$ del total de la muestra. En cuanto a aquellos pacientes que no presentaron síncope en la prueba de mesa basculante, un 59,2\% presentó bostezo de manera temprana con cambios hemodinámicos y del sistema nervioso autónomo significativos, asociado posteriormente a respuesta hemodinámica positiva de la prueba, y al tomar el total de la muestra el bostezo fue predictor de respuesta hemodinámica en casi el $43 \%$ de los pacientes.

\section{Discusión}

El bostezo es una de las conductas humanas más comunes pero menos comprendida. Se trata de un fenómeno motor originado en el tallo cerebral que se asocia con sensación de bienestar ${ }^{10}$. Es filogenéticamente antiguo; se ha documentado en los cinco grupos de vertebrados y tiene funciones adaptativas $^{11}$. En 1873, Charles Darwin describió por primera vez el bostezo, pero fue Barbizet, en 1958, quien lo dividió en tres fases con una duración total entre 4 a 7 segundos ${ }^{11}$ :

Primera fase: apertura lenta y progresiva de la boca, acompañada de expansión de tórax, faringe y laringe junto con descenso del diafragma; aunque la apertura oral y la inspiración profunda también ocurren en otros escenarios, sólo en el bostezo hay expansión marcada de la faringe (tres a cuatro veces lo normal), descenso del hueso hioides y la lengua, y dilatación de la glotis casi hasta su punto máximo.

Segunda fase: apertura máxima de la boca, contracción de los músculos dilatadores de los labios y de los párpados (lo que causa el cierre de los ojos), dilatación de las fosas nasales, máxima dilatación de la faringe e incremento marcado de la capacidad del tórax, secreción de saliva y lágrimas y vasoconstricción en los dedos y taquicardia.

Tercera fase: la inspiración cede súbitamente y la espiración se hace lenta y ruidosa, la faringe vuelve a su tamaño normal, la boca se cierra y los músculos faciales retornan a lo normal.

Son múltiples las hipótesis que han tratado de explicar la función de los bostezos, las dos más aceptadas son las siguientes:

Hipótesis del enfriamiento cerebral: el bostezo es desencadenado por un aumento en la temperatura cerebral para posteriormente retornar a la homeostasis térmica ${ }^{12}$. Se plantea que la inspiración profunda que acompaña al bostezo puede modificar la temperatura de la sangre venosa para drenarla desde los orificios nasales y orales hacia los senos cavernosos ubicados alrededor de la arteria carótida interna, lo cual actúa como un radiador que remueve sangre hipertérmica e introduce sangre arterial más fría al cerebro $^{13,14}$.

Hipótesis del estado de conciencia y vigilia: el bostezo estimula mecánicamente la arteria carótida, lo que promueve un aumento en el estado de alerta cortical por medio de la compresión del bulbo carotídeo y se traduce en la liberación de hormonas como adenosina y catecolaminas ${ }^{15}$. 
Se han desestimado otras explicaciones propuestas como la modificación de los niveles de oxígeno y dióxido de carbono en la sangre ${ }^{16}$ y el bostezo como mecanismo de interacción social ${ }^{17}$.

Al hablar de la relación del bostezo con el sistema nervioso autónomo, los pocos estudios desarrollados a la fecha han demostrado activación del simpático por activación del sistema reticular ubicado en el tallo cerebral relacionado con la red diencéfalo-hipotálamo, similar a lo que sucede con la presión arterial sistólica con la ingesta de cafeína. Se plantea que el bostezo, como la cafeína, pueden desempeñar un rol en el reflejo de alerta. Es así como Corey et al. hallaron un aumento significativo de la frecuencia cardíaca en el pico del bostezo al compararlo con los valores basales $(p<0,001)$ pero con disminución pronta después de 5 segundos y se estableció que esta taquicardia es resultado de la alerta generada por la activación del simpático, aunque no se encontró aumento en la conductancia de la piel (parámetro escogido para medir la actividad del sistema nervioso simpático). Los autores proponen que estos hallazgos sugieren una activación aguda del sistema nervioso simpático con aumento de la frecuencia cardíaca y el volumen tidal y que la disminución de la frecuencia respiratoria que sigue después del bostezo puede indicar relajación posterior a la activación del sistema nervioso simpático; establecen además que tanto el bostezo como la inhalación profunda aumentan la temperatura facial, los volúmenes pulmonares y la frecuencia cardíaca, pero sólo el bostezo produce un aumento transitorio del sistema nervioso simpático. Finalmente, concluyen que el bostezo tiene propósitos fisiológicos como cambios en el estado de alerta y cognitivo, y la temperatura cerebral ${ }^{5}$.

De otra parte, Askenasy et al. demostraron el efecto del bostezo sobre el sistema nervioso simpático. Describieron que los 2 segundos iniciales del bostezo llevan a supresión simpática y disminución media de la presión arterial de 5 a $8 \mathrm{~mm} \mathrm{Hg}$; luego de 3 segundos además se vio que la frecuencia cardíaca no se afectó de manera significativa pero con una tendencia a la bradicardia en el momento del bostezo. En consecuencia, existe una respuesta parasimpática inmediata a los bostezos seguida por una descarga simpática con la espiración final ${ }^{6}$.

Es claro que el síncope neuralmente mediado es precedido por varios signos premonitorios que demuestran estimulación parasimpática: palidez, náuseas, alteraciones visuales y salvas de bostezos, de modo que algunos investigadores plantean que la aparición de bostezos es un signo clínico que debería atraer la atención de los médicos durante la exploración invasiva y permitir la anticipación del síncope ${ }^{18}$.

Para el caso de esta investigación, el principal estímulo que desencadena un bostezo como mecanismo compensador en los pacientes sometidos a prueba de mesa basculante es la hipotensión. El bostezo suele aparecer antes de la administración de nitroglicerina, lo que expresa el agotamiento y disbalance temprano del sistema nervioso autónomo, y predice una respuesta final vasodepresora o mixta.

Este estudio evidenció una disminución significativa de la frecuencia cardíaca con un promedio de 13,7 latidos por minuto que ocurre desde el inicio, predominantemente 5 a 10 segundos posteriores al comienzo del bostezo, hecho que podría explicar la disminución concomitante del índice cardiaco (IC). Este hallazgo es contrario a lo reportado por Corey et al. quienes relacionan al bostezo con taquicardia, similar a los resultados de Askenasy et al. para quienes la frecuencia cardíaca mostró tendencia a la bradicardia. Esta situación probablemente se explica por la sensibilidad barorrefleja (ante un aumento de la presión arterial hay activación de los barorreceptores y disminución consecuente de la frecuencia cardíaca) y por la espiración lenta de la última fase del bostezo que se acompaña de bradicardia, lo cual es similar a lo observado en la fase 4 de la maniobra de Valsalva.

Teniendo en cuenta que la mayoría de pacientes llevados a prueba de mesa basculante son jóvenes sin comorbilidades cardiovasculares mayores, es de esperarse que esta sensibilidad barorrefleja y la disminución correspondiente de la frecuencia cardíaca en respuesta al aumento de la presión arterial (mediado por el tono parasimpático) se encuentren conservadas. Ocurre lo contrario en pacientes con enfermedades cardiovasculares tipo hipertensión arterial, insuficiencia cardíaca o enfermedad coronaria, en quienes existe una activación adrenérgica crónica con reducción de la actividad inhibitoria de los barorreceptores sobre el sistema nervioso simpático y frecuentemente se observa alteración de esta sensibilidad barorrefleja.

La presión arterial, especialmente la presión arterial sistólica, aumentó significativamente en la mayoría de pacientes, en especial al inicio del bostezo, 5 y 10 segundos después, lo cual va de la mano con el aumento estadísticamente significativo de la resistencia vascular periférica.

Al relacionar los hallazgos hemodinámicos con los del sistema nervioso autónomo, se estableció que el bostezo influye directamente con la activación del sistema nervioso simpático reflejado por aumento significativo de la relación LF/HF durante el inicio del mismo al compararlo con el momento previo al evento y a los 15 segundos, además por un incremento de la resistencia vascular periférica, lo que clínicamente se traduce en mejoría de los síntomas, elevación de la presión arterial y, en un porcentaje importante de pacientes, evita que durante el prueba de mesa basculante presenten síncope lo que lo convierte en un mecanismo protector y contrarregulador. En otros casos favorece la compensación temprana de los síntomas y de las variables hemodinámicas sin que el paciente desarrolle síncope o prueba de mesa basculante con respuesta positiva como tal, pero que interpretado en el contexto adecuado se convierte en un signo predictor de disautonomía o prueba de mesa basculante positivo.

\section{Limitaciones del estudio}

La aparición de bostezos tiene un componente de imitación, pues está demostrado que incluso el hablar de bostezos desencadena este fenómeno. Para mitigar este efecto y evitar sesgos, en ningún momento se le mencionó al paciente la palabra bostezo ni que se estaba evaluando este tema.

Se contó con una muestra de 156 pacientes que, aunque no es despreciable, debería ser mayor para poder generar conclusiones que sean más aplicables a la población. Sin embargo, dentro de las fortalezas de esta investigación se destaca que se ha utilizado la cardioimpedancia con medición en tiempo real de variables hemodinámicas y del 
sistema nervioso autónomo que permite una correlación con el bostezo.

\section{Conclusiones}

El bostezo es un fenómeno que se asocia claramente con activación del sistema nervioso simpático y del sistema cardiovascular. Puede plantearse como un signo predictor de respuesta hemodinámica positiva de tipo mixta o vasodepresora, incluso en aquellos pacientes que no presenten síncope ni alcance a ser interpretado como prueba positiva. En otros casos puede proponerse como un mecanismo protector frente a la aparición de síncope.

\section{Financiación}

Ninguna.

\section{Conflicto de intereses}

Ninguno.

\section{Bibliografía}

1. Baron-Esquivias G, Martínez-Alday J, Martín A, Moya A, GarcíaCivera R, Paz López-Chicharro $M$, et al. Epidemiological characteristics and diagnostic approach in patients admitted to the emergency room for transient loss of consciousness: Group for Syncope Study in the Emergency Room (GESINUR) study. Eur Eur Pacing Arrhythm Card Electrophysiol J Work Groups Card Pacing Arrhythm Card Cell Electrophysiol Eur Soc Cardiol. 2010;12:869-76.

2. Hatoum T, Sheldon R. A practical approach to investigation of syncope. Can J Cardiol. 2014;30:671-4.

3. Goldberger JJ. Sympathovagal balance: how should we measure it? Am J Physiol. 1999;276 4 Pt 2:H1273-80.

4. Cooke WH, Rickards CA, Ryan KL, Kuusela TA, Convertino VA. Muscle sympathetic nerve activity during intense lower body negative pressure to presyncope in humans. J Physiol. 2009;587 Pt 20:4987-99.
5. Corey TP, Shoup-Knox ML, Gordis EB, Gallup GGJ. Changes in Physiology before, during, and after Yawning. Front Evol Neurosci [Internet]. 2012. Jan 3;3:7. doi: 10.3389/fnevo.2011.00007. eCollection 2011.

6. Askenasy JJ, Askenasy N. Inhibition of muscle sympathetic nerve activity during yawning. Clin Auton Res Off J Clin Auton Res Soc. 1996;6:237-9.

7. Bartoletti A, Alboni P, Ammirati F, Brignole M, Del Rosso A, Foglia Manzillo G, et al. «The Italian Protocol»: a simplified head-up tilt testing potentiated with oral nitroglycerin to assess patients with unexplained syncope. Eur Eur Pacing Arrhythm Card Electrophysiol J Work Groups Card Pacing Arrhythm Card Cell Electrophysiol Eur Soc Cardiol. 2000;2: 339-42.

8. Sammito S, Böckelmann I. Reference values for time- and frequency-domain heart rate variability measures. Heart Rhythm. 2016;13:1309-16.

9. Askenasy JJ. Is yawning an arousal defense reflex? J Psychol. 1989;123:609-21.

10. Walusinski O. Yawning in diseases. Eur Neurol. 2009;62:180-7.

11. Baenninger R. On yawning and its functions. Psychon Bull Rev. 1997;4:198-207.

12. Gallup GG, Gallup AC. Excessive yawning and thermoregulation: two case histories of chronic, debilitating bouts of yawning. Sleep Breath Schlaf Atm. 2010;14:157-9.

13. Zenker W, Kubik S. Brain cooling in humans-anatomical considerations. Anat Embryol (Berl). 1996;193:1-13.

14. Shoup-Knox ML, Gallup AC, Gallup GG, McNay EC. Yawning and stretching predict brain temperature changes in rats: support for the thermoregulatory hypothesis. Front Evol Neurosci. 2010;2:108.

15. Matikainen J, Elo H. Does yawning increase arousal through mechanical stimulation of the carotid body? Med Hypotheses. 2008;70:488-92.

16. Provine RR, Tate BC, Geldmacher LL. Yawning: no effect of $3-5 \%$ CO2, $100 \%$ O2, and exercise. Behav Neural Biol. 1987;48:382-93.

17. Gallup AC. Why do we yawn? Primitive versus derived features. Neurosci Biobehav Rev. 2011;35:765-9.

18. Cronin TG. Yawning: an early manifestation of vasovagal reflex. AJR Am J Roenitroglicerinaenol. 1988;150:209. 